\title{
Primary Occult Breast Cancer in A Male Patient: A Case Report and Brief Overview of Literature
}

\section{Luis E. Fregoso Arteaga ${ }^{1}$ | Benjamin Zavala Retes ${ }^{1 *} \mid$ Fatima Rincon Cabada $^{1} \mid$ Michael P. White $^{2}$}

\section{*Correspondence: Benjamin Zavala Retes}

Address: ${ }^{1}$ Av. Paseo de la Reforma 476, Juárez, Cuauhtémoc, Oncology Hospital, Mexican institute of Social Security, National Autonomous University of Mexico, 06600, Mexico City, Mexico; 2259 First St, NYU Winthrop Hospital, NYU-Long Island School of Medicine, Mineola, NY 10158, USA

e-mail $\bowtie$ nimajneb_retes@msn.com

Received: 27 January 2021; Accepted: 05 February 2021

Copyright: (C) 2021 Arteaga LEF, et al. This is an open-access article distributed under the terms of the Creative Commons Attribution License, which permits unrestricted use, distribution, and reproduction in any medium, provided the original author and source are credited.

\section{ABSTRACT}

Breast cancer is the most common female cancer, nevertheless it represents a rare entity in male patients; the presentation of breast cancer as a primary occult cancer in male patients is such a rare pathology that only case reports have been described. We present a 48 year old man who developed and axillary mass which resulted to be metastatic breast cancer, he was treated in the same fashion as female patients are treated.

Keywords: Male Breast Cancer, Primary Occult Cancer, Breast Cancer

\section{Introduction}

Breast cancer represents in order of frequency the first cancer worldwide in females with $11.6 \%$ of total. Regarding mortality it accounts for $6.6 \%$ worldwide (followed by lung cancer, colorectal cancer, stomach a liver cancer) (Globocan, 2018; National Cancer Institute, 1975-2011).

Breast cancer in male patients relatively uncommon, $(0.5-1 \%$ of all breast cancer and less than $1 \%$ in male cancer overall). The main risk factors in male population includes genetic mutations such as BRCA 2, CYp17, PTEN and CHECK 2, it is also related to Klinefelter syndrome in 3-7\% of all cases of breast cancer in men (Anderson et al., 2010). Other associated risk factors are consumption of estrogens and testosterone (Nahleh et al., 2007) as well as cryptorchidic testis, obesity, epididymitis and thoracic exposure to radiation. (Fentiman et al. 2006).

Breast cancer is a disease caused by accumulation of multiple genetic aberrations (e.g. mutations, deletions, rearrangements...), $90 \%$ associated with somatic mutations and only $10 \%$ attributed to germline mutations (Bell, 2010). Histologic subtypes may be divided in ductal and lobular carcinoma, being ductal carcinoma the most frequent (about $80 \%$ of all cases); $85 \%$ of patient also features estrogen and 
progesterone receptors (luminal subtypes), only 11\% of patients overexpress HER2 Neu phenotype (Crew et al., 2007; Sasco et al., 1993; Yan-Hui et al., 2016)

The most common clinical presentation of breast cancer in male comprises the growth of a single painless mass in the breast, the skin may be ulcerated, or the nipple retracted in $75 \%$ of the cases (Fentiman et al., 2006)

Nevertheless, the presentation as a primary occult is extremely rare. Primary occult breast cancer is defined as the identification of axillar metastasis compatible with a breast phenotype without clinical or radiological evidence of a breast mass or calcification, it was first described by Halsted; the incidence in female is low ( 0.3 to $0.8 \%$ of all breast cancer), in male there are only case reports that describe the entity (Halsted, 1907; Metin Yalaza et al., 2016; Ruixin et al., 2017).

The diagnostic and therapeutic protocol does not differ from those of female patients; it should be tailored for every patient by a multidisciplinary team; when an axillary tumor is found the initial step is to perform tissue biopsy to determinate malignancy, once this is established the origin of the metastases must be determined by the probability of the origin, in this case breast, thorax, head and neck must be thoroughly inspected in search of a primary tumor; once the diagnosis of primary occult breast cancer the treatment is conducted according to the clinical stage (e.g. surgery followed by adjuvant chemotherapy plus radiotherapy). (Hong et al., 2016; Patten et al., 2013; Wang et al., 2010).

\section{Case Presentation}

A forty-eight-year-old men presented to the office complaining of a progressive growing mass in the left axilla during the last 12 months; in the beginning the patient did not cared about the mass because of the small size and absence of pain, however the mass presented progressive growth and became painful. In physical examination the only finding was a fixed and matted mass in the left axilla measured $5 \times 5 \mathrm{~cm}$, there was only left nipple retraction (Fig. 1) but no clinical evidence of tumor activity in breast, thorax, head, neck, abdomen or inguinal regions. An incisional biopsy was performed in an office setting with pathology report of poorly differentiated metastatic carcinoma.

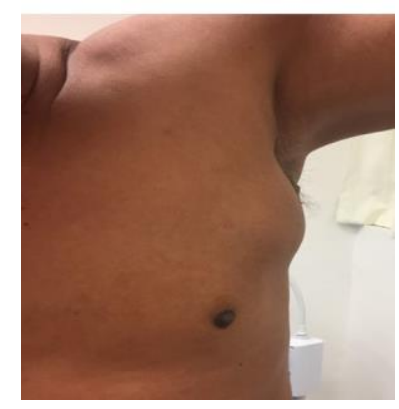

Figure 1: Left axillary matted and fixed nodes 
Immunohistochemistry was performed on tissue (16) with estrogen receptor positive in $90 \%$ of staining, progesterone receptor staining 40\%, PAN CK staining positive, CK positive, GATA 3 positive-intense, GCDFP-1 positive-weak, TTF-1 positive-weak, thyroglobulin positive-weak, CK 20 negative, CK 14 negative, Napsin negative, Her 2 Neu negative (Fig. 2 and Fig. 3).

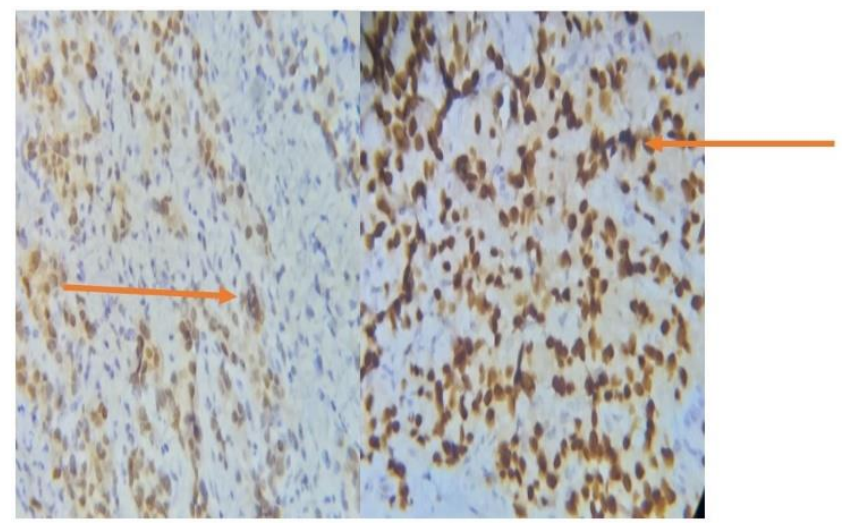

Figures 2 and 3: Immunohistochemistry

Mammography and axillar ultrasound were performed showing normal right axillar nodes and normal right breast; in the left axilla suspicious matted nodes were observed (i.e. irregular form, $4 \times 2.5 \times 1.5 \mathrm{~cm}$, loss of cortex), a left infraclavicular suspicious lymph node was detected (Fig. 4) and sampled for histopathology with positive result for malignancy; the left breast was normal. Mammography for both breasts were reported as BIRADS2 (Breast Imaging Reporting and Data System), the BIRADS system comprises 7 scores ranged from 0 to 6 , each one associated the presence or abscense of anatomic alterations regarding architectural distortion, masses or calcifications, a score of 2 meansbenign changes with no probability of cancer by this metod (American College of Radiology, 2003). A CT scan revealed only tumor activity in left axillar region (Fig. 5 and Fig. 6).

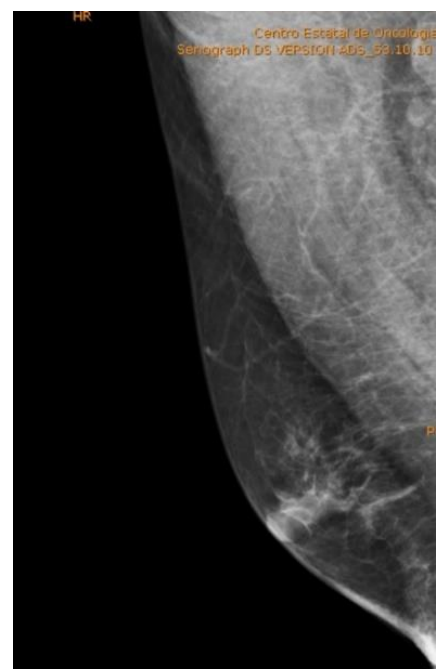

Figure 4: Left breast mammography, BIRADS 2 

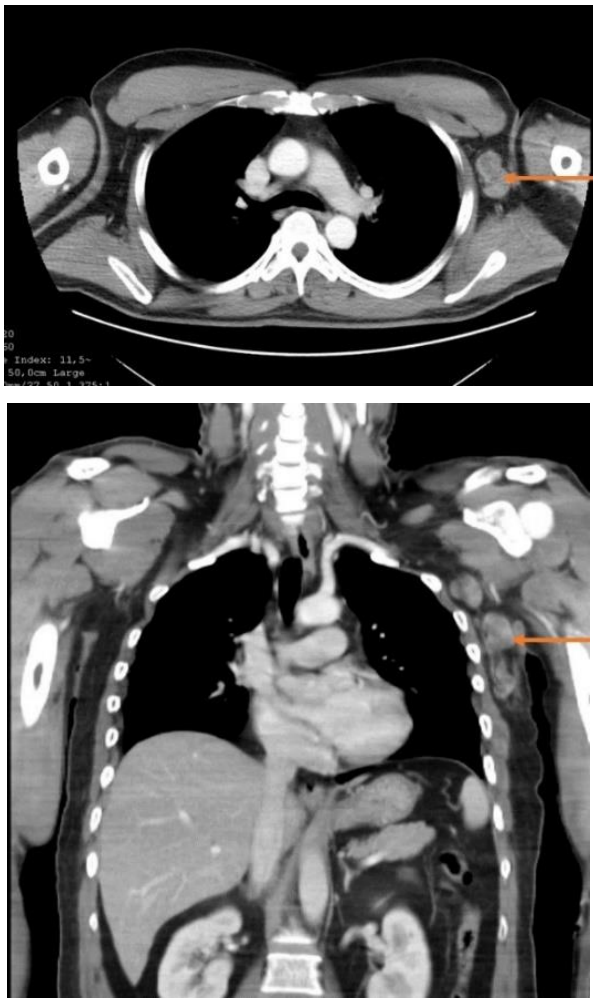

Figures 5 and 6: CT scan showing left axillary matted nodes

The diagnosis of breast cancer in stage IIIC was stablished and the patient went on neoadjuvant treatment with epirubicin followed by cyclophosphamide, clinical partial response was achieved (Fig. 7); the patient went to modified radical left mastectomy with left axillary dissection without complications (Fig. 8 and Fig. 9).

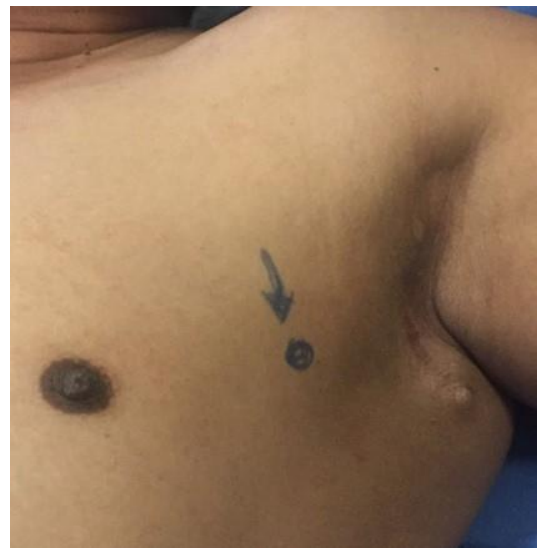

Figure 7: Partial clinical response to neoadjuvant chemotherapy

Pathology report reported absence of tumor in left breast, 10 left axillar nodes were reported with metastatic carcinoma- breast immunophenotype, the nodes were matted measuring $6 \times 6.5 \mathrm{~cm}$, lymphatic, vascular and perineural invasion were present, 5 nodes presented with capsular rupture; skin and nipple had no evidence of tumor. 


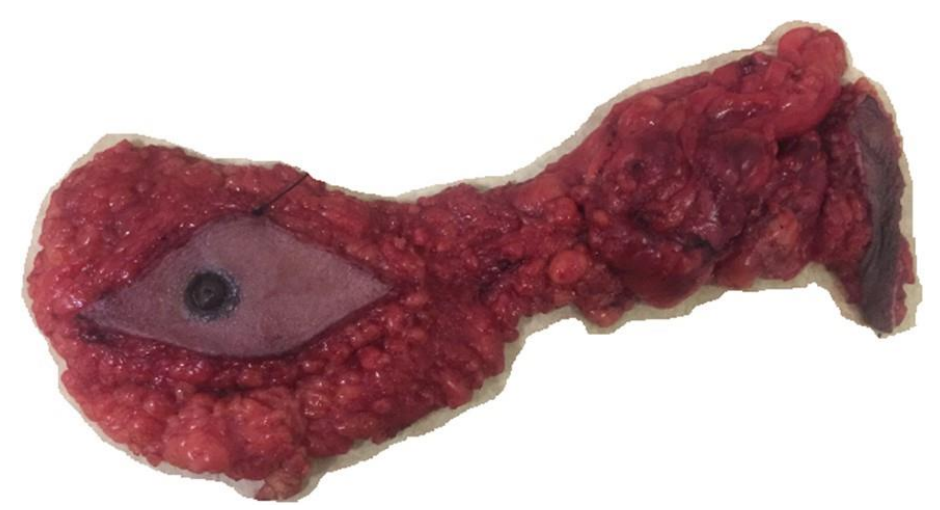

Figure 8: Left radical modified mastectomy including axillary skin from biopsy site

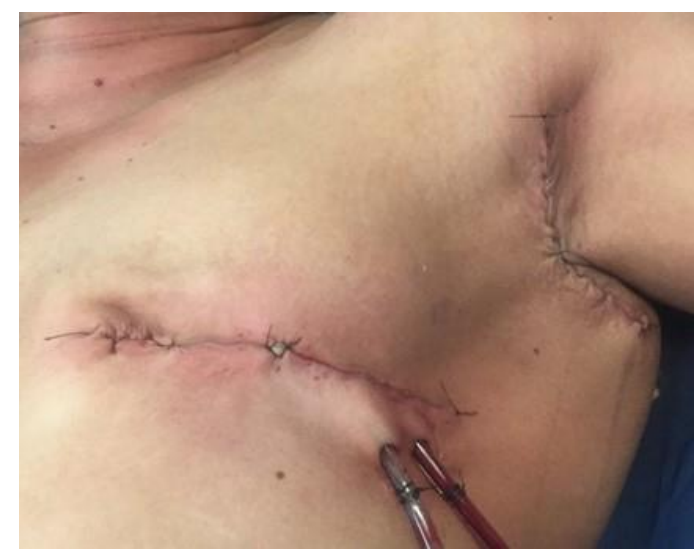

Figure 9: Postoperative surgical site

Patient was given adjuvant treatment with radiation therapy at a dose of 50Gy in 25 fractions (Huang et al., 2004), also tamoxifen was given at a dose of $20 \mathrm{mg}$ PO qd. He has been monitored for 2 years without evidence of recurrence.

\section{Discussion}

Male breast cancer is a very rare pathology that usually represents a diagnostic challenge due to its low prevalence, the risk factors for males are increased exposure to estrogen and reduced androgen, Klinefelter syndrome increases 14- to 50 fold the risk of breast cancer as well as BRCA1 and BRCA 2 mutations (Fentiman et al., 2006; Anderson et al., 2010) clinical presentation of male breast cancer is similar to that of females differing only that men age of onset is a median of 7 years later. the special feature of this case was not only the fact of a male breast cancer but that the clinical presentation corresponded to a primary occult breast cancer; few cases like this have been reported on literature and in all the patients a multidisciplinary approach with chemotherapy, surgery and radiation in neoadjuvant, radical and adjuvant sequence respectively. More than $90 \%$ of these tumors in men are positive to hormonal receptor making necessary to administer hormonal therapy. In this case the patient as survived 2 year free of recurrence and is under oncological surveillance. (Wang et al., 2010; Sousa et al., 2013). 


\section{Conclusion}

Occult primary breast cancer in men is a very uncommon disease that requires high grade of suspicion because the diagnosis is not considered and screen mammography in men is not used; the same considerations regarding diagnostic and treatment as for females are applied, some studies suggest a worse prognosis in males than in females stage by stage.

\section{References}

American College of Radiology. ACR BI-RADS®-Mammography: Breast Imaging Reporting and Data System, Breast Imaging Atlas, vol 4. ACR, Preston, 2003.

Anderson WF, Jatoi I, Tse J, Rosenberg PS. Male breast cancer: a population-based comparison with female breast cancer. J Clin Oncol 2010; 28: 232-239.

Bell DW. Our changing view of the genomic landscape of cancer. J Pathol 2010; 220: 231-243.

Cancer Genome Atlas Network. Comprehensive molecular portraits of human breast tumours. Nature 2012; 490: 61-70.

Crew KD, Neugut AI, Wang X, Jacobson JS, Grann VR, Raptis G, Hershman DL. Racial disparities in treatment and survival of male breast cancer. J Clin Oncol 2007; 25: 1089-1098

Fentiman IS, Fourquet A, Hortobagyi GN. Male breast cancer. Lancet 2006; 367: 595-604.

Globocan. Intrnational angency for Research on Cancer. 2018; Http: //iar.fr/

Halsted WSI. The results of radical operations for the cure of carcinoma of the breast. Ann Surg 1907; 46: 1-19.

Hong JH, Ha KS, Jung YH, Won HS, An HJ, Lee GJ, Kang D, Park JC, Park S, Byun JH, Suh YJ. Clinical features of male breast cancer: experiences from seven institutions over 20 years. Cancer Res Treat 2016; 48: 1389-1398.

Huang EH, Tucker SL, Strom EA, McNeese MD, Kuerer HM, Buzdar AU, Valero V, Perkins GH, Schechter NR, Hunt KK, Sahin AA. Postmastectomy radiation improves local-regional control and survival for selected patients with locally advanced breast cancer treated with neoadjuvant chemotherapy and mastectomy. J Clin Oncol 2004; 22: 4691-4699

Metin Yalaza, Aydın İnan, Mikdat Bozer. Male Breast Cancer. J Breast Health 2016; 12: 1-8

Nahleh ZA, Srikantiah R, Safa M, Jazieh AR, Muhleman A, Komrokji R. Male breast cancer in the veterans affairs population: a comparative analysis. Cancer 2007; 109: 1471-1477.

National Cancer Institute, Surveillance, Epidemiology, and End Results Program SEER Cancer Statistics Review, 19752011.

Patten DK, Sharifi LK, Fazel M. New approaches in the management of male breast cancer. Clin Breast Cancer 2013; 13: 309-314

Ruixin Xu, Jianbin Li, Yingjie Zhang, Hongbiao Jing, Youzhe Zhu. Male occult breast cancer with axillary lymph node metastasis as the first manifestation. Medicine 2017; 96: 51.

Sasco AJ, Lowenfels AB, Pasker-de Jong P. Review article: epidemiology of male breast cancer. A meta-analysis of published case-control studies and discussion of selected aetiological factors. Int J Cancer 1993; 53: 538-549. 
Case Report

DOI: http://dx.doi.org/10.47733/GJCCR.2021.2103

Sousa B, Moser E, Cardoso F. An update on male breast cancer and future directions.

Wang X, Zhao Y, Cao X. Clinical benefits of mastectomy on treatment of occult breast carcinoma presenting axillary metastases. Breast J 2010; 16: 32-37.

Yan-Hui Zhu, Meng-Yao Luo, Zhong Ji, Ju-Feng Guo. Diagnoses and Therapy of Occult Breast Cancer: A Systematic Review. Zhu et al., J Mol Biomark Diagn 2016, S: 2. 